\title{
Los métodos de evaluación de la competencia profesional: la evaluación clínica objetiva estructurada (ECOE)
}

\author{
José María Martínez Carretero \\ Unidad de Evaluación de las Competencias Clínicas. Institut d'Estudis de la Salut (IES). \\ Generalitat de Cataluña.
}

Los sistemas sanitarios, como empresas que prestan servicios a la población, hacen un uso intensivo de recursos humanos altamente cualificados. Si pretendemos que estos servicios sean de calidad, habrá que asegurar la competencia de los profesionales. La evaluación de la competencia clínica es, por lo tanto, un objetivo de las instituciones involucradas en la formación y utilización de los profesionales sanitarios.

Desde hace más de tres décadas diversas instituciones están utilizando métodos docentes y evaluativos de la práctica clínica, por lo cual, disponemos en la actualidad de instrumentos válidos, fiables, aceptables tanto para los candidatos como para las instituciones, factibles y cuyo impacto educativo está demostrado.

La cultura evaluativa de nuestro país ha estado basada fundamentalmente en los métodos tradicionales y muy pocos expertos se han atrevido a valorar, de forma objetiva, la práctica clínica. Desde principios de 1994 se vienen realizando proyectos de evaluación que nos permiten valorar las competencias clínicas a diversos niveles (pregrado, postgrado y en el ejercicio profesional) y de distintos profesionales.

Es necesario considerar la evaluación como un instrumento diagnóstico de los déficits competenciales, y por lo tanto útil para modificar o mejorar la formación previa y posterior, para la selección de los profesionales, para la certificación y recertificación y para cualquier sistema de incentivación y promoción profesional (carrera) que se desee diseñar.

La definición de las competencias de las profesiones es una necesidad obvia, tanto desde el punto de vista de su utilidad docente, de planificación y gestión de los servicios sanitarios, como de la regulación del derecho al ejercicio de la profesión.

Se puede definir competencia como un proceso dinámico y longitudinal en el tiempo, por el cual una persona utiliza los conocimientos, habilidades, actitudes y buen juicio, asociados a su profesión, con la finalidad de poder desarrollarla de forma eficaz en todas las situaciones que corresponden al campo de su práctica. Representa, por tanto, un estado de su praxis profesional.

Las competencias de los profesionales, se pueden clasificar en las siguientes: Asistenciales, las de la Medicina Preventiva y Comunitaria, de investigación y docencia, las habilidades necesarias para relacionarse con otros niveles asistenciales y trabajar en equipo, las éticas y deontológicas y las relacionadas con los conocimientos del entorno sociocultural.

A su vez, las competencias o habilidades asistenciales, se pueden subdividir en: la capacidad para obtener información del paciente, mediante la Historia Clínica y la exploración física, elaborar un informe clínico y comunicarse eficazmente con el paciente y sus familiares, el conocimiento y comprensión de las patologías (conocimientos biomédicos y la interpretación de pruebas complementarias), el juicio clínico y la capacidad para resolver problemas clínicos (priorización de problemas de salud, elaboración de diagnósticos diferenciales y el diseño de planes diagnósticos y terapéuticos), y las habilidades técnicas necesarias para el diagnóstico y el tratamiento (por ejemplo, ser capaz de tomar la tensión arterial o ser capaz de suturar una herida).

La importancia o el peso relativo de cada una de estas capacidades dependerá obviamente de la profesión sanitaria e incluso de la especialización de cada profesión. Numerosas instituciones internacionales y actualmente en nuestro país, relacionadas con la formación y la gestión de los profesionales sanitarios están definiendo estas competencias. 


\section{MÉTODOS DE EVALUACIÓN}

Para evaluar cada una de estas competencias, los instrumentos tienen que ser necesariamente diferentes, dado que no hay ningún método de evaluación que por si solo pueda proporcionar toda la información necesaria para juzgar la competencia de un profesional. Es necesario por tanto, una combinación de los diferentes métodos para evaluar las habilidades cognoscitivas y las complejas habilidades que componen el concepto de competencia profesional.

De acuerdo con la pirámide de Miller hay cuatro niveles de formación por orden de complejidad. En la base de la pirámide están los conocimientos que un profesional necesita saber para desarrollar sus tareas profesionales con eficacia, en el nivel superior estará la capacidad para saber como utilizar estos conocimientos para analizar e interpretar los datos obtenidos. Esta capacidad se define como competencia. No tan solo es preciso conocer o saber como utilizar sino también es necesario demostrar como se utilizan. Es decir, es necesario conocer la actuación de un profesional frente a una situación clínica específica. Finalmente, no obstante, es preciso conocer lo que un profesional hace realmente en su práctica laboral. (Figura 1)

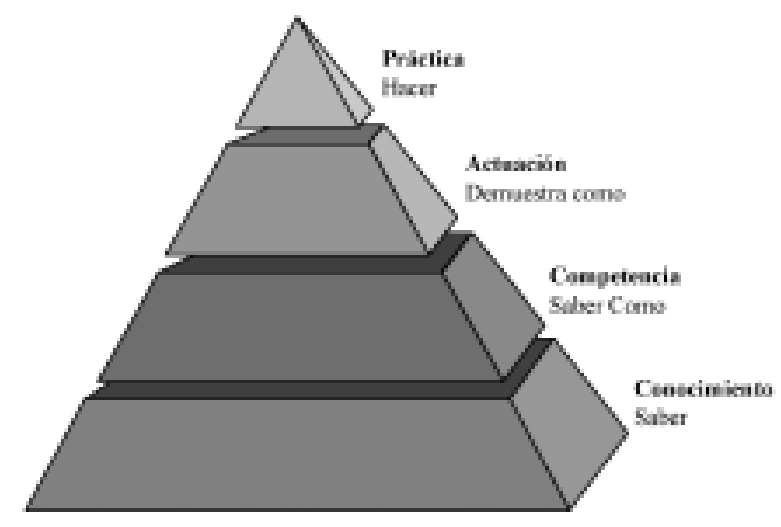

Pirámide de Miller

Fig. 1

\section{Métodos para evaluar los conocimientos}

Las preguntas escritas y especialmente las preguntas de elección múltiple, han sido las más empleadas porque son más validas, fiables y fáciles de elaborar, que otros métodos y pueden proporcionar un amplio abanico de información, sobre las habilidades cognoscitivas, incluidas las habilidades para interpretar pruebas complementarias.

\section{Métodos para evaluar la competencia}

Los métodos empleados para evaluar competencia incluyen los clásicos exámenes orales y las pruebas escritas cortas o largas. Con estos métodos se puede evaluar la capacidad teórica para resolver problemas clínicos de los pacientes, así, la simulación escrita de un caso clínico es un buen instrumento para evaluar esta habilidad.

\section{Métodos para evaluar la actuación}

Los métodos utilizados para evaluar este nivel de la pirámide de Miller, se basan fundamentalmente en las simulaciones, que intentan reproducir situaciones similares de la vida real en condiciones estandarizadas, que permiten que los observadores puedan analizar las actuaciones específicas que se pretenden evaluar.

Estos métodos incluyen, las simulaciones por ordenador y los enfermos simulados estandarizados. El paciente simulado estandarizado constituye uno de los instrumentos educativos y evaluativos más importantes para garantizar que se dispone de las competencias clínicas necesarias en el encuentro médico-paciente. Los pacientes simulados son individuos especialmente entrenados para cumplir dos funciones: representar una situación clínica determinada de acuerdo a una patología previamente establecida y evaluar la capacidad del profesional en la obtención de una anamnesis adecuada, en la exploración física y en los patrones de comunicación con el paciente. Dado que todos estos pacientes son entrenados, se comportan, responden y evalúan de una manera uniforme con todos los evaluados, se les considera estandarizados.

A pesar de la importancia y utilidad del paciente simulado estandarizado, con propósitos ya sea docentes o evaluativos de unas áreas específicas de competencias clínicas, es imprescindible la combinación con otros instrumentos que nos permitan evaluar otras competencias clínicas.

Una mención especial merece la prueba conocida como OSCE (Objective Structured Clinical Examination) o Evaluación Clínica Objetiva y Estructurada (ECOE).

La ECOE es un formato de prueba en el que se pueden incluir diferentes métodos evaluativos. El formato básico consiste en que los candidatos roten por un circuito de estaciones secuenciales en el que se les solicita que realicen una variedad de diferentes habilidades. En muchas de estas estaciones se utilizan Pacientes Simulados estandarizados, casos por ordenador, maniquíes, pruebas complementa- 


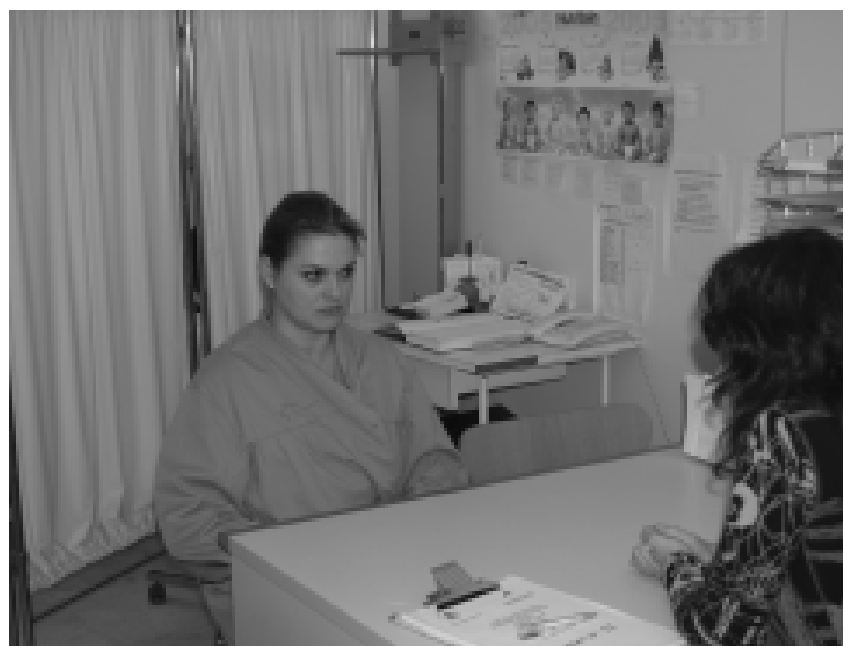

Fig. 2

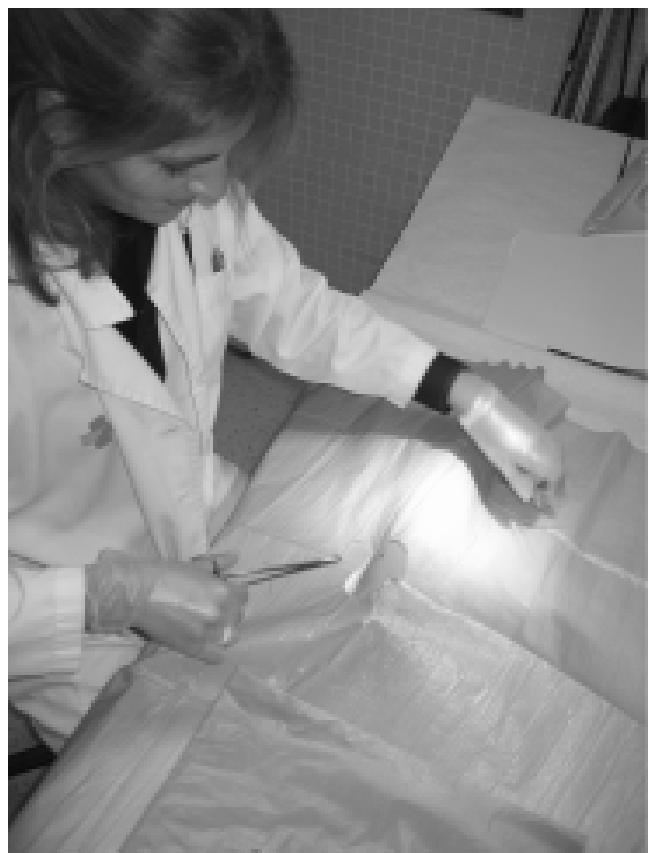

Fig. 3

rias (ECG, RX, analítica, etc), preguntas de respuesta múltiple o corta relacionadas con los casos, entre otras. (Figuras 2 y 3 ).

Para que la ECOE sea válida y fiable debe reunir, de acuerdo con la literatura científica, una serie de condiciones o características: La duración debe ser entre 3 y 4 horas, tener 8 o más pacientes simulados, cada estación debe durar 10 minutos, tener un máximo de 30 ítems de evaluación por caso, no más de 20 candidatos a evaluar en cada sesión y combinar, de acuerdo con las competencias a evaluar, varios instrumentos evaluativos como los mencionados, entre otras.

\section{Métodos para evaluar la práctica profesional}

Es obvio que este nivel de evaluación es el más importante y completo, ya que nos proporciona información sobre lo que el profesional realmente hace en su práctica profesional. No obstante, es preciso señalar, que desde el punto de vista metodológico es el más difícil ya que intervienen no solamente problemas técnicos (de factibilidad y fiabilidad) sino también otros factores no relacionados con lo que el profesional es capaz de hacer y que pueden modificar su práctica, como por ejemplo, el tipo de organización de la institución en la que trabaja, los recursos disponibles, la competencia de otros profesionales que intervienen en la práctica que se pretende evaluar, la masificación asistencial y la motivación del propio profesional, entre otras.

A pesar de todo, existen instrumentos que pretenden evaluar este nivel y nos pueden dar información bastante relevante de la práctica real del profesional como: las escalas de evaluación global, la revisión de las historias clínicas (audit), la revisión de las decisiones clínicas (chart stimulated recall), las observaciones de la práctica por colegas o mediante videos, las encuestas de satisfacción de enfermos y familiares, cuadernos de autoaprendizaje o portafolio, la opinión de otros miembros del equipo, los indicadores de problemas en la práctica, o la calidad de las prescripciones terapéuticas, entre otras.

\section{La valoración formativa}

Los métodos antes señalados pueden ser utilizados como instrumentos de evaluación del progreso competencial durante el proceso de formación con fines fundamentalmente formativos y no exclusivamente sumativos tanto en el pregrado como en el postgrado. La información obtenida, a nivel individual o grupal, permite mejorar y adaptar los métodos de aprendizaje así como los contenidos al progreso de los estudiantes. La valoración formativa es un instrumento imprescindible en las entrevistas tutor-estudiante/residente (feed back) de la formación basada en la tutorización activa continua.

\section{NIVELES DE EVALUACIÓN}

$\mathrm{Al}$ menos se pueden distinguir tres niveles diferentes de evaluación en el desarrollo profesional continuo individual: Al final de la formación pregraduada, al finalizar la formación postgraduada con propósitos de certificación de la especialidad y 


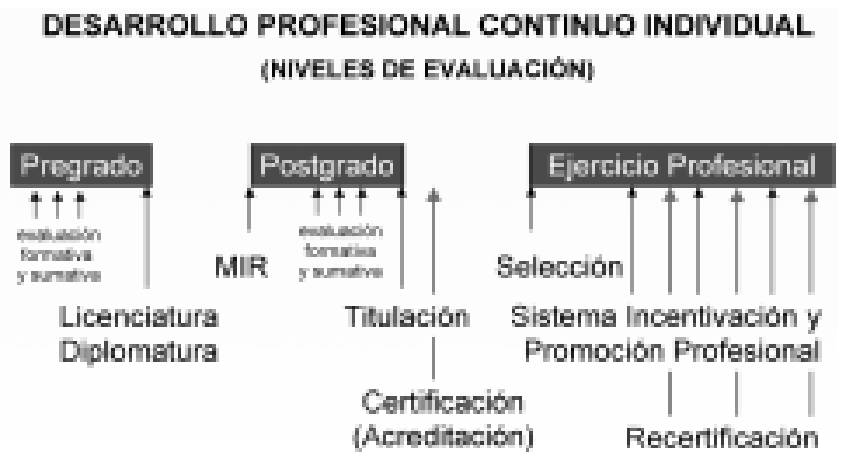

- Definición de competencias

- Metodos abjetivos de evaluación

Fig. 4

durante el trabajo independiente de los profesionales con el propósito de recertificación y carrera profesional. (Figura 4)

Será necesario por tanto dirigir acciones evaluadoras a estos tres niveles, que serán necesariamente diferentes, no tan solo en cuanto a contenidos sino a los métodos a utilizar. Además, será necesario utilizar los métodos de evaluación objetiva para la selección de los profesionales para adecuarlos a sus lugares de trabajo.

Por lo que respecta a las instituciones responsables que tendrían que utilizar los métodos de evaluación en los niveles antes mencionados tendrían, a nuestro entender, de ser en el pregrado, las Facultades de Medicina y Escuelas Universitarias de Enfermería conjuntamente con los colegios profesionales, que son los organismos responsables de garantizar a la sociedad la competencia de sus profesionales. En el nivel de especialización serían las sociedades científicas como organismos que agrupan a los diferentes especialistas. En la selección de los profesionales, de acuerdo con las necesidades explicitadas por la definición de las competencias para un lugar de trabajo determinado, los centros sanitarios (proveedores sanitarios). Finalmente, la recertificación sería responsabilidad de las sociedades científicas y/o de los colegios profesionales y la carrera profesional o sistema de incentivación y promoción profesional correspondería a cada centro sanitario donde el profesional ejerce.

En un país como el nuestro, en el cual no hay tradición de evaluación de los profesionales al finalizar la formación postgraduada (certificación de la especialización) y/o durante el ejercicio profesional (recertificación), con frecuencia se confunden, los objetivos, contenidos y métodos de eva- luación de estos con los de un sistema de desarrollo profesional.

De acuerdo con las experiencias internacionales, a menudo representada por los países anglosajones (USA, Australia y Canadá), la certificación es necesario entenderla como la evaluación que se realiza al finalizar una formación especializada, con el objetivo de garantizar que se han adquirido aquellos conocimientos, habilidades y actitudes que conforman la especialidad. Se trata por tanto de evaluar un abanico amplio de competencias de la especialidad y los métodos de evaluación, cada vez más, se basan en simulaciones tipo OSCE. Las instituciones responsables de garantizar estas competencias son las sociedades científicas (Boards).

Sobre la recertificación, se trataría de evaluar las competencias comunes a todos los profesionales de una especialidad determinada, independientemente de la subespecialidad o experteza que esté desarrollando actualmente, se le evaluaría periódicamente sobre las competencias "nucleares" de la especialidad. Los instrumentos de evaluación empleados conjugan las baremaciones de actividades formativas, las evaluaciones tipo OSCE y otros métodos de evaluación de la práctica real.

Por tanto, el concepto de carrera / promoción-desarrollo profesional no está relacionado ni con la certificación ni con la recertificación profesional. En todo caso, estas últimas serian requisitos previos a partir de los cuales seria preciso evaluar la experteza y/o el cumplimiento de los objetivos asistenciales que constituyen el contenido de cualquier sistema de desarrollo profesional.

Cualquier sistema de incentivación y promoción profesional ha de contemplar estos contenidos y los métodos de evaluación se deben basar en las tareas reales de los profesionales.

En las dos últimas conferencias de Educación Médica, la 11th International Ottawa Conference on Medical Education (Barcelona 6-8 de Julio de 2004) y en el Congreso de la AMEE (Association for Medical Education in Europe, Edimburgo 5-8 de Septiembre de 2004) se han tratado ampliamente los aspectos conceptuales, los métodos e instrumentos para la evaluación del desarrollo profesional continuado individual.

\section{EXPERIENCIAS EN CATALUÑA SOBRE EVALUACIÓN DE LA COMPETENCIA}

En Cataluña, el Institut d'Estudis de la Salut inició en 1994, de acuerdo con estas directrices y orien- 


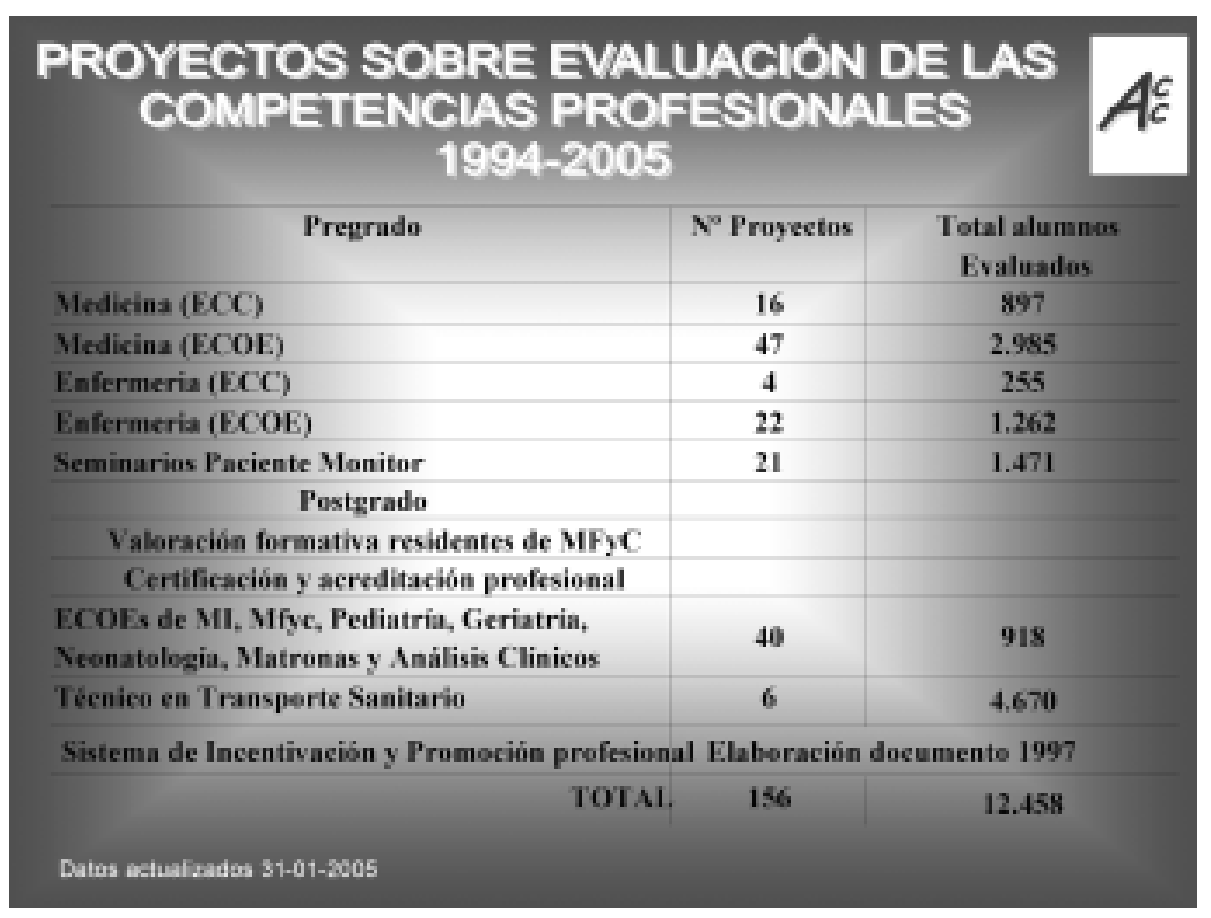

Fig. 5

taciones internacionales, diferentes proyectos de evaluación al final de la formación de pregrado de médicos y enfermeras, en el postgrado con la valoración formativa de los residentes de la especialidad de Medicina familiar y comunitaria, para la certificación profesional con diversas sociedades científicas y colaboró en la elaboración de un documento, finalizado en 1997, sobre las bases conceptuales para un sistema de incentivación y promoción profesional que ha servido de guía para los convenios y acuerdos de las mesas de negociación de las patronales sanitarias y los sindicatos (Convenio de la Red Hospitalaria de utilización pública. RHUP 2001-
2004 y Acuerdo de la mesa sectorial del Instituto Catalán de la Salud. ICS 2002-2006). (Figura 5)

El Libro Blanco de las profesiones sanitarias de Cataluña, publicado el año pasado, contempla muchos de los aspectos básicos mencionados sobre el desarrollo profesional continuo individual y ahora se está procediendo al desarrollo operativo con diferentes grupos de trabajo, uno de ellos vinculado específicamente a la evaluación y reconocimiento profesional.

Cabe señalar que, a partir de 1998 estos proyectos evaluativos adoptaron el formato evaluativo tipo ECOE (Evaluación Clínica Objetiva y Estructurada), que permitía explorar todas las Competencias Clínicas. 